International Journal of Pure and Applied Mathematics

Volume 84 No. 5 2013, 593-611

ISSN: 1311-8080 (printed version); ISSN: 1314-3395 (on-line version)

url: http://www.ijpam.eu

doi: http://dx.doi.org/10.12732/ijpam.v84i5.12

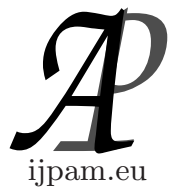

\title{
EXISTENCE OF PERIODIC WAVE SOLUTION IN SPRING-BLOCK MODEL WITH SLIP-DEPENDENT FRICTION
}

\author{
Kodwo Annan \\ Department of Mathematics \& Computer Science \\ Minot State University \\ Minot, North Dakota, 58707, USA
}

\begin{abstract}
The global conditions under which a spring-block model coupled with non smooth slip-dependent friction force depicts a seismic fault are studied. Existence and uniqueness conditions of the periodic orbits solutions were proved using Poincare-Bendixsom theorem and Lyapounov-Schmidt reduction technique. The insight gained has important implications for the understanding of earthquakes and other dissipative driven systems.
\end{abstract}

\section{AMS Subject Classification: 86A17}

Key Words: spring-block, earthquakes, friction, slip-dependent, existence

\section{Introduction}

Earthquakes display a rich set of complex behaviors, and a wealth of quantitative data exists describing these behaviors. Yet the origin of these behaviors remains largely unexplained, and finding a set of well behaved mathematical equations which can reproduce all the behaviors is still an as yet unachieved goal. However, simple spatially extended dynamical models have shown that a remarkably rich set of earthquake-like behaviors can arise from slip-dependent

Received: December 19, 2012

(c) 2013 Academic Publications, Ltd. url: www.acadpubl.eu 
non-smooth friction applied to the spring-block models [1]. It is generally believed that one of the basic mechanisms of earthquakes is frictional instability arising at the interface of tectonic plates [2]. This viewpoint is supported by the manifestation of static-dynamic friction duality causing the jerking motion that often occurs when two surfaces are sliding over each other (also known as the stick-slip phenomenon [3]).

A mathematical description of the stick-slip motion is a long-standing problem in dynamical systems, since it requires a description of the dynamics at very different spatial and temporal scales, ranging from microscopic interactions between two surfaces to the macroscopic nonlinear dynamics of the moving bodies [4-8]. Attempts to reduce the complexity in the theory of frictional dynamics resulted in the introduction of the spring-block models, which reproduce well the basic features of the frictional phenomena and do not contradict observational results. The basic idea [4] underlying this class of models includes the mechanism of contacts to the constitutive "friction law", while considering the effects of inertia and deformations through the introduction of masses and springs [5-8]. The aforementioned models are further complicated by some variants of non-smooth friction laws and model parameters that make it highly difficult to obtain solution [9].

Since the introduction of the spring-block model for fault mechanisms many similar models have been proposed which purportedly generate earthquake moments [10-13]. Carlson and Langer [12], for example, demonstrated that block arrays subjected to a simple velocity weakening friction reproduced the Gutenberg-Richter scaling law between the magnitude of earthquakes and their occurrence rate [13]. The periodic solution generated from their model was spatially homogeneous with initial conditions. However, any spatial in-homogeneity fault model caused by variable non-smooth friction during the slip may affect the characteristics of the traveling waves.

In this paper, we apply the velocity-weakening friction law introduced by Carlson and Langer to prove the existence of a periodic orbit and solution generated by spring-block model. The proof for the non-smooth uncoupled model was based on Poincare-Bendixsom theorem and is similar to the one presented in [14]. Since the non-smoothness in the slip-dependent friction added more coupled differential equation to the model system, we deployed LyapounovSchmidt reduction technique to show that there exists a periodic solution for the weakly coupled spring-block model system.

The paper is presented as follows: We state the model problem, notation and preliminaries in Section 2. We then prove the existence of a periodic solution for non-smooth uncoupled and coupled versions of the model in Section 3. 
Conclusion is given in Section 4.

\section{Problem, Notation and Preliminaries}

\subsection{Problem Statement}

We state here the dimensionless non-smooth equation for a spring-block periodic wave solution $x_{i}$ in the moving blocks as [14]

$$
\frac{\ddot{x}_{i}}{\tau^{2}}=\ell^{2}\left(x_{i+1}-2 x_{i}+x_{i-1}\right)-x_{i}-F\left(V+\frac{\dot{x}_{i}}{\tau}\right) .
$$

Here the spring stiffness ratio $\ell$, the non-linear velocity-dependent friction force $F$, and the weakening velocity strength $V$ are all dimensionless parameters and defined in $[14,15]$. Equation (1) reveals that the model characteristics is controlled by those three dimensionless parameters with the traveling wave velocity of $\tau^{-1}$. The existence and uniqueness of the periodic solution for (1) was proved for uncoupled and coupled problems with smoothed friction force [14]. We now prove the existence and uniqueness of (1) with non-smooth friction force for both uncoupled $(\ell=0)$ and coupled $(\ell \neq 0)$ at any value of $\tau=\tau_{0} \in \mathbb{R}^{+}$.

\subsection{Notation and Preliminaries}

A function $x:[0, T] \rightarrow \mathbb{R}^{n}$ is absolutely continuous if given $\varepsilon>0$, there exists $\delta>0$ such that for any countable collection of disjoint subintervals $\left[t_{n}, t_{n}^{\prime}\right]$ of $[0, T]$ satisfying $\sum\left(t_{n}^{\prime}-t_{n}\right)<\delta$ whenever $\sum\left|x\left(t_{n}^{\prime}\right)-x\left(t_{n}\right)\right|<\varepsilon$.

Definition 2.1. Consider a differential inclusion of order one

$$
\dot{x}(t) \in F(x(t)), t \in[0, T],
$$

where $F: \mathbb{R}^{n} \rightarrow \mathbb{R}^{n}$ is a set-valued map. The solution to (2) is continuous absolutely and satisfies (2) almost everywhere in the time interval $[0, T]$.

Definition 2.2. Let $x \in C^{1}(\mathbb{R})$ be a T-periodic function and piecewise in $C^{2}(\mathbb{R})$. Then $x$ is a periodic solution of (1) if it satisfies almost everywhere in $[0, T]$.

Since $x \in C^{1}$, it is Lischitz continuous and easy to use the mean value theorem to show that it is continuous absolutely. Also, the derivative $\dot{x}$ is piecewise in $C^{2}$. Suppose that there exists a point of discontinuity $t_{0} \in\left[t_{n}, t_{n}^{\prime}\right]$, then $f_{-}=\dot{x}_{\left|\left[t_{n}, t_{0}\right)\right|} \in C^{1}$ which can be extended to $\left[t_{n}, t_{0}\right]$ by the $C^{1}$ function 
$\tilde{f}_{-}$. Similarly, $f_{+}=\dot{x}_{\left.\mid] t_{0}, t_{n}^{\prime}\right] \mid}$ in $\left.] t_{0}, t_{n}^{\prime}\right]$ is a $C^{1}$ function $\left(\tilde{f}_{+} \in\left[t_{0}, t_{n}^{\prime}\right]\right)$ that can be extended. Thus,

$$
\begin{aligned}
& f\left(t_{n}\right)-f\left(t_{0}\right)=\tilde{f}_{-}\left(t_{n}\right)-\tilde{f}_{-}\left(t_{0}\right)=\int_{t_{n}}^{t_{0}} \tilde{f}_{-}^{\prime}(t) d t=\int_{\left|t_{n}, t_{0}\right|} f_{-}, \\
& f\left(t_{0}\right)-f\left(t_{n}^{\prime}\right)=\tilde{f}_{+}\left(t_{0}\right)-\tilde{f}_{+}\left(t_{n}^{\prime}\right)=\int_{t_{0}}^{t_{n}^{\prime}} \tilde{f}_{+}^{\prime}(t) d t=\int_{\left|t_{0}, t_{n}^{\prime}\right|} f_{+}^{\prime} .
\end{aligned}
$$

Defining $g_{n}=f_{-}^{\prime} \in\left[t_{n}, t_{0}\left[, g_{n}=f_{+}^{\prime} \in\right] t_{0}, t_{n}^{\prime}\right]$ and, $g_{n}\left(t_{0}\right)=0$, in a $C_{m}^{0}$ function, we have

$$
\begin{gathered}
f\left(t_{n}\right)-f\left(t_{n}^{\prime}\right)=f\left(t_{n}\right)-f\left(t_{0}\right)+f\left(t_{0}\right)-f\left(t_{n}^{\prime}\right) \\
=\int_{\left[t_{n}, t_{0}[\right.} g_{n}+\int_{] t_{0}, t_{n}^{\prime}\right]} g_{n}=\int_{\left[t_{n}, t_{n}^{\prime}\right]} g_{n} \\
\Rightarrow\left|f\left(t_{n}\right)-f\left(t_{n}^{\prime}\right)\right| \leq\left\|g_{n}\right\|_{\infty,\left[t_{n}, t_{n}^{\prime}\right]}\left|t_{n}-t_{n}^{\prime}\right| \leq \sup _{k}\left\|g_{n}\right\|_{\infty,\left[t_{n}, t_{n}^{\prime}\right]}\left|t_{n}-t_{n}^{\prime}\right| .
\end{gathered}
$$

Thus, $f=\dot{x}$ is absolutely continuous.

Theorem 2.3. For all $V>0$, there exists a periodic orbit to equation (1).

Proof. The proof is based on Poincare-Bendixson theorem which is proved in $[14]$.

However, we assume that there exists a periodic orbit given by theorem 2.3 of the form:

$$
\dot{x}(t)=-\tau V \text { in }\left[0, t_{g}\right] \text { and } \dot{x}(t) \neq-\tau V \text { in }\left[t_{g}, T_{0}\right],
$$

This means that in an interval $\left[0, t_{g}\right]$, the masses stick to the lower surface of the spring-block model while at $t=t_{g}$, when the strain threshold is exceeded, they begin to move.

\section{Global Existence of Periodic Solutions}

\subsection{Existence of Uncoupled Non-Smooth Problem}

We now prove the existence of the periodic solution for the uncoupled nonsmooth version of equation (1) for any value of $\tau=\tau_{0} \in \mathbb{R}^{+}$by setting $\ell=0$, scale time as $t \tau$, and define a non-smooth friction force $F$. Thus (1) becomes

$$
\ddot{x}+x+F(V+\dot{x})=0 .
$$


Proposition 3.1. The inclusion equation (6) has at least one solution on $\mathbb{R}^{+}$for all initial conditions.

Proof. The differential $\dot{X}(t) \in G(X)$, where $G: \mathbb{R}^{2} \rightarrow P\left(\mathbb{R}^{2}\right) \backslash \Phi\left(P\left(\mathbb{R}^{2}\right)\right)$. We have existence of an absolute continuous solution on $\mathbb{R}^{+}$. If the multivalued function, $G$, is a closed convex, upper semi-continuous and does not grow too fast then there exists a constant $c>0$ such that for all $X \in \mathbb{R}^{2}$ and $z \in G(X)$, we have $\|z\| \leq c(1+\|X\|)$. So, the second dimensional form of (6) in first order can be written as

$$
\dot{X} \in G(X) \text {, where } X=\left(\begin{array}{c}
x \\
\dot{x}
\end{array}\right) ; G(X)=\left(\begin{array}{c}
\dot{x} \\
-x
\end{array}\right)+\left(\begin{array}{c}
0 \\
-F(V+\dot{x})
\end{array}\right) .
$$

Clearly, $F$ is a closed convex in $\mathbb{R}^{2}$ and so $G$ is also a closed convex in $\mathbb{R}^{2}$. The function $G$ is upper semi-continuous if for all $A$ closed in $\mathbb{R}^{2}$, we have $G^{-1}(A)$ also closed in $\mathbb{R}^{2}$. This is correspondent to showing that $F$ is an upper semi-continuous. If we define a segment $[a, b]$ in $\mathbb{R}$, then $F^{-1}([a, b])$ is of the form $\Phi,\{0\} \cup[\alpha, \beta],\{0\} \cup[\alpha,+\infty[,\{0\} \cup]-\infty, \beta]$ or $\{0\} \cup]-\infty, \beta] \cup[\alpha,+\infty[$. Hence, $F^{-1}([a, b])$ is closed and $F$ is upper semi-continuous. In addition we can write

$$
\begin{aligned}
\|G(X)\|_{2} \leq\left\|\left(\begin{array}{c}
\dot{x} \\
-x
\end{array}\right)\right\|_{2}+\left\|\left(\begin{array}{c}
0 \\
-F(V+\dot{x})
\end{array}\right)\right\|_{2} \\
\quad \leq\|X\|_{2}+\sup _{y}\left\|\left(\begin{array}{c}
0 \\
F(y)
\end{array}\right)\right\|_{2} \leq\|X\|_{2}+M,
\end{aligned}
$$

Since $F$ is bounded, the not too fast growing condition is satisfied. Thus, we have existence of a solution for all initial conditions

Proposition 3.2. There exists a unique solution for the inclusion equation (6) in $C^{1}$ and piecewise in $C^{2}$.

Proof. By phase plot analysis illustrated in Figure 1, clearly, as long as $x$ is a solution of $\dot{x}(t)=-V$, the right-hand-side of (6) is perfectly smooth. Thus, by Cauchy-Lipschitz theorem the solution is unique. To verify that the two different solutions cannot pass through a point $\left(x_{0},-V\right)$, we define a vector field $\nu(x, \dot{x})=\vec{\nu}=\left(\begin{array}{c}\dot{x} \\ -x-F(V+\dot{x})\end{array}\right)$. Then

$$
\lim _{(x, \dot{x}) \rightarrow\left(x_{0},-V^{+}\right)} \vec{\nu}=\left(\begin{array}{c}
-V \\
-x_{0}-F_{0}
\end{array}\right) \text { and } \lim _{(x, \dot{x}) \rightarrow\left(x_{0},-V^{-}\right)} \vec{\nu}=\left(\begin{array}{c}
-V \\
-x_{0}+F_{0}
\end{array}\right) .
$$




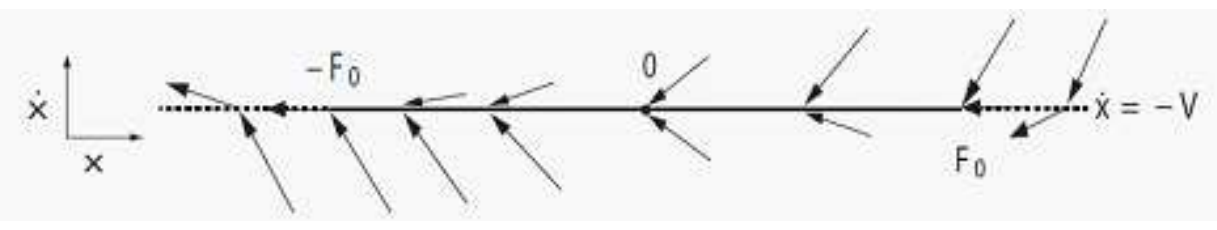

Figure 1: Phase space plot for $\dot{x}=-V$.

Therefore, as $x \rightarrow\left(x_{0},-V\right)$ with $\left|x_{0}\right|>F_{0}$, we have the two solutions crossing the line $\dot{x}=-V$. However, if $x \rightarrow\left(x_{0},-V\right)$ with $\left|x_{0}\right| \leq F_{0}$, the solution $x$ cannot cross the segment $\left[-F_{0}, F_{0}\right] \times\{-V\}$ since it is contractive (see phase space below). In addition, since $x$ and $\dot{x}$ are continuous, $\ddot{x}$ is also continuous except when $\dot{x} \rightarrow-V$ (i.e. at the end of the sliding period). Thus, $\ddot{x}$ is piecewise in class $C^{2}$.

Lemma 3.3. Let $L(t)=(x(t), \dot{x}(t))$ be a trajectory of (6) and $X_{\text {eq }}$ defines a unique equilibrium point. Then the distance $D\left(L(t), X_{e q}\right)$ is increasing in the half plane $y>-V$.

Proof. We define $X_{e q}=(-F(V), 0)$ so that $D\left(L(t), X_{e q}\right)^{2}=(x(t)+F(V))^{2}+$ $\dot{x}^{2}$. This implies

$$
\frac{d}{d x}\left[D\left(L, X_{e q}\right)^{2}\right](t)=2 \dot{x}[\ddot{x}+x+F(V)]
$$

Implying

$$
\begin{aligned}
\frac{d}{d t}\left[D\left(L, X_{e q}\right)^{2}\right](t)>0 \Leftrightarrow 2 \dot{x}[-x-F(V+\dot{x})+x+F(V)]>0, & \\
& \Leftrightarrow \dot{x}[F(V)-F(V+\dot{x})]>0 .
\end{aligned}
$$

Assume that $\dot{x}>0$,then $F(y)=\frac{F_{0}}{1+y}$ for all $y>0$. Thus, $F$ is decreasing in $\mathbb{R}^{+}$ and $F(V)>F(V+\dot{x})$. Hence $D\left(L, X_{e q}\right)$ is increasing. If $-V<\dot{x}<0$, then since $V+\dot{x}>0$, it implies $F(V+\dot{x})>F(V)$ and consequently $\dot{x}[F(V)-F(V+$ $\dot{x})]>0$. Therefore, $D\left(L(t), X_{e q}\right)$ is also increasing.

Proposition 3.4. Suppose that $x_{0}$ is a T-periodic solution of (6) of the form

$$
\left\{\begin{array}{l}
\dot{x}_{0}(t)=-V \text { for }\left[0, t_{g 0}\right] \\
\dot{x}_{0}(t) \neq-V \text { for }\left[t_{g 0}, T\right] .
\end{array}\right.
$$

Then any trajectory of (6) with initial values into domain delimited by $X_{0}=$ $\left(x_{0}, \dot{x}_{0}\right)$ reaches the periodic orbit in finite time. 


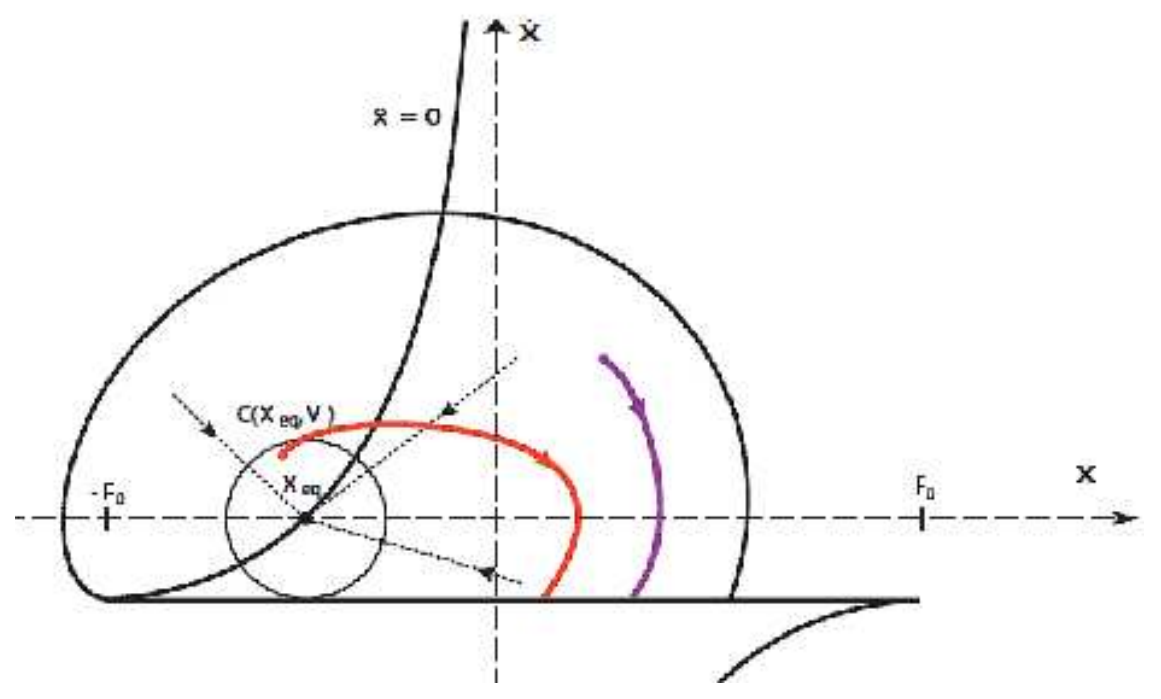

Figure 2: Semi-attractive plot of the periodic solution.

Proof. We consider initial conditions in the domain delimited by $X_{0}$ which is outside of the disk of radius $V$ and center $X_{e q}$, as shown in Figure 2. Since the disk is tangent to the segment $\left[-F_{0}, F_{0}\right] \times\{-V\}$, it follows from Lemma 3.3 that the trajectory must cross the segment in finite time. If we locate an initial point in the disk of radius $V$ and center $X_{e q}$, we finds that the distance to $X_{e q}$ is strictly increasing. Suppose then that the trajectory does not travel outside the disk, then there is no attractive equilibrium point into the disk and so the trajectory converges to a limit cycle. Since the trajectory distance is increasing, the limit cycle is a circle. However, the distance to $X_{e q}$ is constant in the case of a circle trajectory which contradicts the lemma. Thus, every trajectory with initial point into the disk must go out of the disk.

Proposition 3.5. If $X_{0}=\left(x_{0}(t), \dot{x}_{0}(t)\right)$ is a periodic orbit passing through the point $\left(-F_{0},-V\right)$, then there is no other periodic orbit in the domain delimited by the orbit $X_{0}$.

Proof. Suppose there exists a periodic orbit with initial condition into the domain delimited by the orbit $X_{0}$. Then from Proposition 3.4 it reached $\left(x_{0}(t), \dot{x}_{0}(t)\right)$ in finite time. Therefore, it would not be a periodic orbit unless it is $X_{0}$. 
Remark 3.6. Therefore, the existence of the periodic orbit for the uncoupled non-smooth model follow the Poincare-Bendixson property proved in [14]. Now, it is necessary to determine the conditions under which it is unique by considering two periodic orbits as follow:

- Case 1: When $V$ is smaller than $F_{0}$, the periodic trajectories start at the point $\left(-F_{0},-V\right)$ and then crosses again the line $\dot{x}=-V$ at the point $(y,-V)$ such that $-F_{0}<y<F_{0}$.

- Case 2: When for large $V$, the periodic trajectories crosses the line $\dot{x}=-V$ at the point $(y,-V)$ such that $y>F_{0}$.

For both cases, the trajectory orbits are in $C^{1}$ and piecewise in $C^{2}$. Therefore, from propositions 3.4 and 3.5 the periodic orbits generated by cases 1 and 2 are semi-attractive with no other periodic orbits inside them.

\subsection{Existence of Coupled Non-Smooth Problem}

Again assuming that $x_{0}$ defined in (13) is a $T_{0}$-periodic solution for $\tau=\tau_{0}$ that describes a stick-slip characteristic for $t \in\left[0, t_{g 0}\right]$.

$$
\left\{\begin{array}{l}
\dot{x}_{0}(t)=-\tau_{0} V \text { for }\left[0, t_{g 0}\right], \\
\dot{x}_{0}(t) \neq-\tau_{0} V \text { for }\left[t_{g 0}, T_{0}\right] .
\end{array}\right.
$$

Thus, equation (13) indicates that at time $t \in\left[0, t_{g}\right]$, the masses stick to the lower surface while at time $t \approx t_{g}=t_{g 0}$ the masses begin to move. We now show the existence of periodic solution $x$ of (1) traveling at speed $\tau^{-1}>0$ when $\ell$ is close to zero by first stating the following hypotheses and theorem.

\section{Hypotheses 14.}
(i) $T_{0} \gg 1$;
(ii) $T_{0}-t_{g 0} \ll 1$;
(iii) $x_{0}$ is Case 1 Type.

Theorem 3.7. Assume that $x_{0}$ is a $T_{0}$-periodic solution of the form (13) for $\tau=\tau_{0} \in \mathbb{R}^{+}, x_{0}(0)=x_{0}\left(T_{0}\right)<F_{0}$ of (6) and satisfying the hypotheses (14). Let denote $t_{g 0}$ as time at which the mass begins to slide and $x_{0 i}$ for $i \in\{1,2\}$ as two linearly independent solutions on the time interval $] t_{g 0}, T_{0}[$ with initial conditions $x_{01}\left(t_{g 0}\right)=1, \dot{x}_{01}\left(t_{g 0}\right)=0, x_{02}\left(t_{g 0}\right)=0, \dot{x}_{02}\left(t_{g 0}\right)=1$, for the equation

$$
\tau_{0}^{-2} \ddot{x}_{0 i}+x_{0 i}+F^{\prime}\left(V+\tau_{0}^{-1} \dot{x}_{0}\right) \tau_{0}^{-1} \dot{x}_{0 i}=0 ; \quad i \in\{1,2\} .
$$


If we represent (15) by the solution $x_{p, \hbar}$

$$
\left.\tau_{0}^{-2} \ddot{x}+x+F^{\prime}\left(V+\tau_{0}^{-1} \dot{x}_{0}\right) \tau_{0}^{-1} \dot{x}=\hbar, \quad t \in\right] t_{g 0}, T_{0}[,
$$

where $\hbar=2 \ddot{x}_{0}+\tau_{0} F^{\prime}\left(V+\tau_{0}^{-1} \dot{x}_{0}\right) \dot{x}_{0}$ and $x_{p, \hbar}\left(t_{g 0}\right)=\dot{x}_{p, \hbar}\left(t_{g 0}\right)=0$ and we further assume that

$$
-\tau_{0} V \dot{x}_{02}\left(T_{0}\right)+\tau_{0} V+\tau_{p, \hbar}^{-2}\left(T_{0}\right) \neq 0 ; \text { for } x_{02}\left(t_{g 0}\right)=0 ; \dot{x}_{02}\left(t_{g 0}\right)=1 .
$$

Then, there exists a neighborhood $\psi$ of $0 \in \mathbb{R}$, and a neighborhood $\Omega$ of $\left(x_{0}, \tau_{0}, t_{g 0}\right)$ in $H^{2}\left(0, t_{g 0}\right) \times\left(\mathbb{R}^{+}\right)^{2}$ such that $\forall \ell \in \psi$, the solution

$$
\left(x(\ell), \tau(\ell), t_{g}(\ell)\right) \in \Omega,
$$

satisfies (1) with $\tau=\tau(\ell)$.

Proof. For $t \in] t_{g 0}, T_{0}[$, we are looking for periodic orbits of (1) in the form of (13) within $\left(t_{g}, T_{0}\right)$ corresponding to when the masses slide. Thus, $\left(t_{g}, T_{0}\right)$ for (1) is no more inclusion and $t_{g}$ is unknown. Defining $x_{1}$ as a small perturbation depending on parameter $\ell$, equation (1) is a differential equation with $x \in C^{1}(\mathbb{R})$ and piecewise in $C^{2}(\mathbb{R})$. Therefore, in order to use the LyapounovSchmidt reduction to find $x=x_{0}+x_{1}$ on $\left.t \in\right] t_{g 0}, T_{0}$ [, we need to satisfy the compatibility condition (16) in Theorem (3.7) and show that the sum of all external forces equals the threshold $\left(-F_{0}\right)$ when the system begins to slip. That is,

$$
\begin{aligned}
& -F_{0}=x\left(t_{g}\right)-\ell^{2}\left(x\left(t_{g}-1\right)-2 x\left(t_{g}\right)+x\left(t_{g}+1\right)\right), \\
& \quad=\left(1+2 \ell^{2}\right) x\left(t_{g}\right)-\ell^{2}\left(-2 \tau V t_{g}+\tau V T_{0}+2 x(0)\right), \\
& \quad=\left(1+2 \ell^{2}\right)\left(-\tau V t_{g}+x(0)\right)-\ell^{2}\left(-2 \tau V t_{g}+\tau V T_{0}+2 x(0)\right), \\
& \quad=-\tau V t_{g}-\ell^{2} \tau V T_{0}+x(0) \\
& \Rightarrow x(0)=-F_{0}+\tau V t_{g}+\ell^{2} \tau V T_{0}
\end{aligned}
$$

Therefore, we have

$$
\begin{aligned}
x(t) & =-\tau V t-F_{0}+\tau V t_{g}+\ell^{2} \tau V T_{0} \\
& =\tau V\left(t_{g}-t\right)-F_{0}+\ell^{2} \tau V T_{0} \text { for } t \in\left[0, t_{g}\right] .
\end{aligned}
$$

So far we have shown that we can perform a Lyapounov-Schmidt reduction to determine the unknowns $\tau, t_{g}$ and $x$ in $] t_{g}, T_{0}$ [provided $(i)$.$x is a T_{0}$-periodic solution of (1), (ii.) there exists $\tau$ and $t_{g}$ such that $x$ is of the form (13), (iii.) $t_{g} \approx t_{g 0}$ and $\tau \approx \tau_{0},(i v$.$) equation (17) is true and (v.) \|x\|_{\infty} \leq 2\left\|x_{0}\right\|_{\infty}$.

Lemma 3.8. The periodic solution, $x$, given by (17) is the solution of (1) in the time interval $\left[t_{g}, T_{0}\right]$ under the hypotheses (14). 
Proof. We first prove that $x$ is a solution of $(1)$ in $\left[0, t_{g}\right]$. Whent $\left.\left.\in\right] 0, t_{g}\right]$, we have $\ddot{x}=0$, thus, equation (1) becomes

$$
x(t)-\ell^{2}(\underbrace{x(t+1)-2 x(t)+x(t-1)}_{W}) \in-F_{0} .
$$

Given that $x$ is continuous, (18) holds for all $t \in\left[0, t_{g}\right]$. To compute the term $W$ in $\left.t \in] 0, t_{g}\right]$, we already know that $x(t)$ is given in (17). However, $t-1$ and $t+1$ may not be in $\left.t \in] 0, t_{g}\right]$. So, we split the interval for $\left.\left.t \in\right] 0, t_{g}\right]$ as $\left.t+1 \in] 1, t_{g}+1\right]$ into three subintervals as

- SUBINTERVAL 1: $\left.\left.\left.t+1 \in] 1, t_{g}\right] \Rightarrow t \in\right] 0, t_{g}-1\right]$,

- SUBINTERVAL 2: $t+1 \in\left[t_{g}, T_{0}\right] \Rightarrow t \in\left[t_{g}-1, T_{0}-1\right]$,

- SUBINTERVAL 3: $t+1 \in\left[T_{0}, t_{g}+1\right] \Rightarrow t \in\left[T_{0}-1, t_{g}\right]$.

Similarly, $\left.t-1 \in]-1, t_{g}-1\right]$ gives

- SUBINTERVAL 4: $\left.\left.\left.t-1 \in]-1,-\left(T_{0}-t_{g 0}\right)\right] \Rightarrow t \in\right] 0,1-\left(T_{0}-t_{g 0}\right)\right]$,

- SUBINTERVAL 5: $t-1 \in\left[-\left(T_{0}-t_{g 0}\right), 0\right] \Rightarrow t \in\left[1-\left(T_{0}-t_{g 0}\right), 1\right]$,

- SUBINTERVAL 6: $t-1 \in\left[0, t_{g}-1\right] \Rightarrow t \in\left[1, t_{g}\right]$.

Only subintervals 2 and 5 remain unknown. The subintervals $1,3,4$ and 6 can be given by explicit formula. Regrouping the common subintervals for both $\left.t+1 \in] 1, t_{g}+1\right]$ and $\left.\left.t-1 \in\right]-1, t_{g}-1\right]$ produces five distinct subintervals (DS) which we will analyze separately below.

DS 1 and 6: $t \in\left[1, t_{g}-1\right] \Rightarrow t-1, t, t+1 \in\left[0, t_{g}\right]$.

Since (17) is satisfied for $x(t-1), x(t)$, and $x(t+1)$, (18) becomes

$$
\begin{aligned}
& x(t)=-\tau V t+x(0) \in\left[-F_{0}, F\right], \quad \forall t \in\left[1, t_{g}-1\right] \\
\Leftrightarrow & \tau V\left(t_{g}-t\right)-F_{0}+\ell^{2} \tau V T_{0} \in\left[-F_{0}, F\right], \quad \forall t \in\left[1, t_{g}-1\right] \\
\Leftrightarrow & 0 \leq \tau V\left(t_{g}-t\right)-F_{0}+\ell^{2} \tau V T_{0} \in\left[-F_{0}, F\right], \quad \forall t \in\left[1, t_{g}-1\right]
\end{aligned}
$$

Since $\tau, V, T_{0} \geq 0$ and $t_{g}-t \geq 0$ in $\left[1, t_{g}-1\right]$, it follows that the lower inequality of (19) is satisfied. Furthermore for small $\ell$, since $\tau V t_{g}$ is very close to $\tau_{0} V t_{g 0}$ the upper inequality of (19) is satisfied. Hence, the last equation of (19) is satisfied.

DS 2 and 6: $t \in\left[t_{g}-1, T_{0}-1[\Rightarrow t-1, t \in] 0, t_{g}\right]$, and $t+1 \in\left[t_{g}, T_{0}[\right.$. 
From (17), we have $x(t)=-\tau V t+x(0)$ and $x(t-1)=-\tau V t(t-1)+x(0)$ explicitly. However, $x(t+1)$ is unknown. Thus,

$$
\begin{aligned}
& x(t+1)-2 x(t)+x(t-1)=x(t+1)+\tau V t+\tau V-x(0) \\
\Rightarrow & x(t)-\ell^{2}(x(t+1)-2 x(t)+x(t-1)) \in\left[-F_{0}, F_{0}\right] \\
\Leftrightarrow & \left|-\tau V t+x(0)-\ell^{2}(x(t+1)+\tau V t+\tau V-x(0))\right| \leq F_{0}
\end{aligned}
$$

Therefore,

$$
0 \leq \tau V\left(t_{g}-t\right)+\ell^{2} \tau V T_{0}-\ell^{2}(x(t+1)+\tau V t+\tau V-x(0)) \leq 2 F_{0} .
$$

Since $\|x\|_{\infty} \leq 2\left\|x_{0}\right\|_{\infty}$, it follows that $|x(t+1)+\tau V t+\tau V-x(0)| \leq 4\left\|x_{0}\right\|_{\infty}+$ $\tau V T_{0}+\tau V=\mathrm{H}$. We need to show that the following inequalities are satisfied in order to conclude (20).

$$
\begin{aligned}
& 0 \leq \tau V\left(t_{g}-t\right)+\ell^{2} \tau V T_{0}-\ell^{2} \mathrm{H}, \quad \text { at } t=T_{0}-1, \\
& \tau V\left(t_{g}-t\right)+\ell^{2} \tau V T_{0}-\ell^{2} \mathrm{H} \leq 2 F_{0}, \quad \text { at } t=t_{g}-1 .
\end{aligned}
$$

From $t_{g} \approx t_{g 0}$ and $\tau \approx \tau_{0}$ and using the hypotheses (14), we have for $t=T_{0}-1$ and $t=t_{g}-1$ respectively,

$$
\begin{aligned}
& \tau V\left(t_{g}-t\right)+\ell^{2} \tau V T_{0}-\ell^{2} \mathrm{H}=\tau V\left(1-\left(T_{0}-T_{g}\right)+\ell^{2} T_{0}\right)-\ell^{2} \mathrm{H} \geq 0 \\
& \tau V\left(t_{g}-t\right)+\ell^{2} \tau V T_{0}-\ell^{2} \mathrm{H}=\tau V+\ell^{2} \tau V T_{0}+\ell^{2} \mathrm{H} .
\end{aligned}
$$

Since $t_{g}-T_{0} \ll 1$, we deduce that $1-\left(T_{0}-t_{g}\right)+\ell^{2} T_{0}>0$ when $\ell$ is close to zero. Also for $\ell \approx 0$ and $\left|\tau-\tau_{0}\right| \rightarrow 0$, we have $\tau V+\ell^{2} \tau V T_{0}+\ell^{2} \mathrm{H} \leq 2 F_{0}$. Thus, (20) is satisfied for small $\ell$.

DS 3 and 6: $\left.\left.t \in\left[T_{0}-1, t_{g}\right] \Rightarrow t-1, t \in\right] 0, t_{g}\right]$, and $t+1 \in\left[T_{0}, T_{0}+t_{g}\right]$. In this case $x(t-1), x(t)$, and $x(t+1)$ are given explicitly as $x(t)=-\tau V t+x(0)$, $x(t-1)=-\tau V(t-1)+x(0)$ and $x(t+1)=-\tau V\left(t+1-T_{0}\right)+x(0)$ which deduce to

$$
\begin{aligned}
& x(t+1)-2 x(t)+x(t-1)=\tau V T_{0}, \\
& x(t)-\ell^{2}(x(t+1)-2 x(t)+x(t-1))=\tau V\left(t_{g}-t\right)-F_{0} .
\end{aligned}
$$

Hence $x$ is a solution of (1) if and only if

$$
0 \leq \tau V\left(t_{g}-t\right) \leq 2 F_{0}, \quad \forall t \in\left[T_{0}-1, t_{g}\right] .
$$

Clearly $\tau V\left(t_{g}-t\right) \geq 0$. Moreover, since $\tau V\left(T_{0}-t_{g}\right) \geq 0$ and $t_{g 0} \gg 1$ (based on hypotheses (14)) we have $\tau_{0} V \ll 2 F_{0}$. Thus, $\tau V \ll 2 F_{0}$ for small $\ell$ and $\left|\tau-\tau_{0}\right| \rightarrow 0$. So $(21)$ is satisfied. 
DS 1 and 4: $\left.\left.\left.t \in] 0,1-\left(T_{0}-t_{g 0}\right)\right] \Rightarrow t, t+1 \in\right] 0, t_{g}\right]$, and $t-1 \in\left[-1,-T_{0}+\right.$ $\left.t_{g}\right] \subset\left[-T_{0},-T_{0}+t_{g}\right]$.

This means, $x(t)=-\tau V t+x(0), x(t-1)=-\tau V\left(t-1+T_{0}\right)+x(0)$ and $x(t+1)=-\tau V(t+1)+x(0)$, and so

$$
\begin{aligned}
& x(t+1)-2 x(t)+x(t-1)=\tau V T_{0}, \\
& x(t)-\ell^{2}(x(t+1)-2 x(t)+x(t-1))=\tau V\left(t_{g}-t\right)-F_{0}+2 \ell^{2} \tau V T_{0} .
\end{aligned}
$$

Implying

$$
\begin{aligned}
x(t) & +\ell^{2} \tau V T_{0} \in\left[-F_{0}, F_{0}\right] \\
& \left.\left.\Leftrightarrow \tau V\left(t_{g}-t\right)+2 \ell^{2} \tau V T_{0} \leq 2 F_{0} \quad \forall t \in\right] 0,1-\left(T_{0}-t_{g 0}\right)\right] .
\end{aligned}
$$

Clearly, for $\ell$ small the inequalities are satisfied at $t=0$ under hypotheses (14).

DS 1 and 5:t $\in\left[1-\left(T_{0}-t_{g 0}, 1\right] \Rightarrow t-1 \in\left[-T_{0}+t_{g}, 0\right]\right.$. Thus, $t, t+1 \in$ ] $\left.0, t_{g}\right]$ and $t-1 \in\left[-T_{0}+t_{g}, 0\right]$.

Similar to DS 2 and 6 we have $x(t)+\ell^{2} \tau V T_{0} \in\left[-F_{0}, F_{0}\right] \Leftrightarrow$

$$
0 \leq \tau V\left(t_{g}-t\right)+\ell^{2} \tau V T_{0}-\ell^{2}\left(\tau V(t-1)-x(0)+x(t-1) \leq 2 F_{0},\right.
$$

which satisfies in $\left[1-\left(T_{0}-t_{g 0}\right), 1\right]$ for small $\ell$ and concludes the prove for the lemma.

We now construct the solution of (1) in the time interval $x \in\left[t_{g}, T_{0}\right]$ and prove that it is a $T_{0}$-periodic solution when $x \in C^{1}$ and piecewise in $C^{2}$.

Let define a function $\bar{x}(k)=x(t)$ for $k \in\left[t_{g 0}, T_{0}\right]$ and rescale time by stating that $k(t)=m t+b$ then for $k\left(t_{g}\right)=t_{g 0}$ and $k\left(T_{0}\right)=T_{0}$ so that we can compare $x_{0}$ and $x$ in the same interval, we have

$$
\begin{aligned}
& m^{2}\left(t_{g}\right) \tau^{-2} \ddot{\bar{x}}(k)+\bar{x}(k)+F\left(V+m\left(t_{g}\right) \tau^{-1} \dot{\bar{x}}\right) \\
& =\ell^{2}\left(-2 m^{-1} \tau V k-2 \bar{x}(k)+C\left(\tau, t_{g}, \ell\right)\right.
\end{aligned}
$$

where $m=m\left(t_{g}\right)=\frac{t_{g 0}-T_{0}}{t_{g}-T_{0}}, b=b\left(t_{g}\right)=T_{0} \frac{t_{g 0}-t_{g}}{T_{0}-t_{g}}$ and

$$
C\left(\tau, t_{g}, \ell\right)=\ell^{2}\left(2 m^{-1} V b+\tau V T_{0}+2 x(0)\right) .
$$

In addition $\bar{x}$ must be in $C^{1}$ such that the following four conditions are satisfied.

$$
\begin{gathered}
\bar{x}\left(t_{g 0}\right)=\ell^{2} \tau V T_{0}, \\
\dot{\bar{x}}\left(t_{g 0}\right)=\tau_{0} V-m^{-1} \tau V,
\end{gathered}
$$




$$
\begin{gathered}
\bar{x}\left(T_{0}\right)=-\tau_{0} V t_{g 0}+\tau V t_{g}+\ell^{2} \tau V T_{0}, \\
\dot{\bar{x}}\left(T_{0}\right)=\tau_{0} V-m^{-1} \tau V .
\end{gathered}
$$

Thus, (24) reduces to

$$
\begin{aligned}
& m^{2} \tau^{-2}\left(\ddot{\bar{x}}+\ddot{x}_{0}\right)+\bar{x}+x_{0}+F\left(V+m \tau^{-1}\left(\dot{\bar{x}}+\dot{x}_{0}\right)\right) \\
& =\ell^{2}\left(-2 m^{-1} \tau V k-2 \bar{x}-2 x_{0}\right)+C\left(\tau, t_{g}, \ell\right) .
\end{aligned}
$$

Lemma 3.9. (Applying Lyapounov-Schmidt Reduction)

For all $\ell \approx 0$, equation (29) has $T_{0}$-periodic solution $\bar{x}$ satisfying conditions (25)-(28) for $\tau$ and $t_{g}$.

Proof. Let represent (29) and its reliability conditions by $g: C^{2}(] t_{g 0}, T_{0}[) \times$ $\mathbb{R}^{3} \rightarrow C^{0}(] t_{g 0}, T_{0}[) \times \mathbb{R}^{4}$ written as $q\left(\bar{x}, \tau, t_{g}, \ell\right)$

$$
=\left\{\begin{array}{c}
m^{2} \tau^{-2}\left(\ddot{\bar{x}}+\ddot{x}_{0}\right)+\bar{x}+x_{0}+F\left(V+m \tau^{-1}\left(\dot{\bar{x}}+\dot{x}_{0}\right)\right) \\
-\ell^{2}\left(-2 m^{-1} \tau V k-2 \bar{x}-2 x_{0}\right)+C\left(\tau, t_{g}, \ell\right) \\
\text { for } \bar{x}\left(t_{g 0}^{+}\right)-\ell^{2} \tau V T_{0}, \quad \dot{\bar{x}}\left(t_{g 0}^{+}\right)-m^{-1} \tau V-\tau_{0} V, \\
\bar{x}\left(T_{0}^{-}\right)+\tau_{0} V t_{g 0}-\tau V t_{g}-\ell^{2} \tau V T_{0}, \quad \dot{\bar{x}}\left(T_{0}^{-}\right)+m^{-1} \tau V-\tau_{0} V,
\end{array}\right.
$$

Then, we have to solve

$$
q\left(\bar{x}, \tau, t_{g 0}, \ell\right)=0 \text { for } q\left(0, \tau_{0}, t_{g 0}, 0\right)=0 .
$$

Proposition 3.10. Let define a linear operator, $L$, by the linear part of $q$ as $L(\bar{x})=D_{\bar{x}} q\left(0, \tau_{0}, t_{g 0}, 0\right) \cdot \bar{x}$ at $m\left(t_{g 0}\right)=1$, where $\bar{x} \in C^{2}\left(\left[t_{g 0}, T_{0}[\right.\right.$, then the kernel of $L$ is zero, i.e. $\operatorname{ker} L=\{0\}$.

Proof. Since $m\left(t_{g 0}\right)=1$ for $\bar{x} \in C^{2}\left(\left[t_{g 0}, T_{0}[\right.\right.$, we have

$$
L(\bar{x})=\left\{\begin{array}{cc}
\tau_{0}^{-2} \ddot{\bar{x}}+\bar{x}+F^{\prime}\left(V+\tau_{0}^{-1} \dot{x}_{0}\right) \cdot \tau_{0}^{-1} \dot{\bar{x}} \\
\bar{x}\left(t_{g 0}^{+}\right), \quad \overline{\bar{x}}\left(t_{g 0}^{+}\right), \quad \bar{x}\left(T_{0}^{-}\right), \quad \overline{\dot{x}}\left(T_{0}^{-}\right)
\end{array}\right.
$$

Suppose that $\bar{x}\left(t_{g 0}^{+}\right)=\lim _{t \rightarrow t_{g 0}^{+}} \bar{x}(t)=0$ and $\bar{x}\left(T_{0}^{-}\right)=\lim _{t \rightarrow T_{0}^{-}} \bar{x}(t)=0$, then $\bar{x} \equiv 0$ since $F^{\prime}\left(V+\tau_{0}^{-1} \dot{x}_{0}\right)$ results to finite limits when $t \rightarrow t_{g 0}^{-}$and $t \rightarrow T_{0}^{+}$. We now extend $F^{\prime}\left(V+\tau_{0}^{-1} \dot{x}_{0}\right)$ under $\left[t_{g 0}, T_{0}\right]$ and claim that $\hat{x}$ is a unique solution of (31) with initial condition $\hat{x}(t)=A$ and $\dot{\hat{x}}(t)=B$. Thus, $\hat{x}_{\mid] t_{g 0}, T_{0}[\mid}=\bar{x}$. Since $\hat{x}$ is $C^{1}$ under $\left[t_{g 0}, T_{0}\right]$ and both $\bar{x}$ and $\dot{\hat{x}}$ identical to zero at $t_{g 0}$ and $T_{0}$, we have $\hat{x}\left(t_{g 0}\right)=\hat{x}\left(T_{0}\right)=\dot{\hat{x}}\left(t_{g 0}\right)=\dot{\hat{x}}\left(T_{0}\right)=0$. Thus $\hat{x}=\bar{x} \equiv 0$. 
Now, representing the non-linear form of $(30)$ by $L(\bar{x})=\lambda\left(\bar{x}, \tau, t_{g}, \ell\right)$, we have $\lambda\left(\bar{x}, \tau, t_{g}, \ell\right)$

$$
=\left\{\begin{array}{c}
\left(\tau_{0}^{-2}-m^{2} \tau^{-2}\right) \ddot{\bar{x}}-m^{2} \tau^{-2} \ddot{x}_{0}-x_{0}+\ell^{2}\left(-2 m^{-1} \tau V k-2 \bar{x}-2 x_{0}\right) \\
+C\left(\tau, t_{g}, \ell\right)-F\left(V+m \tau^{-1}\left(\dot{\bar{x}}+\dot{x}_{0}\right)\right)+F^{\prime}\left(V+\tau_{0}^{-1} \dot{x}_{0}\right) \cdot \tau_{0}^{-1} \dot{\bar{x}} \\
\ell^{2} \tau V T_{0}, V\left(\tau_{0}-m^{-1} \tau\right), V\left(\tau t_{g}-\tau_{0} t_{g 0}\right)+\ell^{2} \tau V T_{0}, V\left(\tau_{0}-m^{-1} \tau\right)
\end{array} .\right.
$$

Projecting $\Pi$ onto the range of $L$ gives

$$
\begin{gathered}
L(\bar{x})=\Pi \lambda\left(\bar{x}, \tau, t_{g}, \ell\right), \\
\Rightarrow \quad(I d-\Pi) \lambda\left(\bar{x}, \tau, t_{g}, \ell\right)=0 .
\end{gathered}
$$

Since $L$ is bijective from $C^{2}\left(\left[t_{g 0}, T_{0}\right]\right) \rightarrow \mathbb{R}(L)$, we can solve (32) using the Implicit Function Theorem. However, (33) is not easy to solve for $\tau$ and $t_{g}$ as a function of $\ell$. Let define an equivalent of (33) as

$$
\varphi_{1}\left(\lambda^{*}\left(\tau, t_{g}, \ell\right)\right)=\varphi_{2}\left(\lambda^{*}\left(\tau, t_{g}, \ell\right)\right)=0
$$

where $\lambda^{*}\left(\tau, t_{g}, \ell\right)=\lambda\left(\bar{x}^{*}\left(\tau, t_{g}, \ell\right), \tau, t_{g}, \ell\right)$ and $\varphi_{1}, \varphi_{2}$ are given by

$$
\left\{\begin{array}{l}
\varphi_{1}(\eta, \bar{\alpha})=\alpha_{2} x_{02}\left(T_{0}\right)-\alpha_{3}+x_{p, \eta}\left(T_{0}\right)+\alpha_{1} \\
\varphi_{2}(\eta, \bar{\alpha})=\alpha_{2} \dot{x}_{02}\left(T_{0}\right)-\alpha_{4}+\dot{x}_{p, \eta}\left(T_{0}\right)+\alpha_{1} \dot{x}_{01}\left(T_{0}\right) \\
\text { for }(\eta, \bar{\alpha})=\left(\eta,\left(\alpha_{1}, \alpha_{2}, \alpha_{3}, \alpha_{4}\right)\right) \in R(L) \\
\text { and } x_{p, \eta}(t)=c_{1}(t) x_{01}(t)+c_{2}(t) x_{02}(t) ; \dot{c}_{1} x_{01}+\dot{c}_{2} x_{02}=0
\end{array}\right.
$$

and define $\Phi: \mathbb{R}^{3} \rightarrow \mathbb{R}^{2}$ given by $\Phi\left(\tau, t_{g}, \ell\right)=\varphi_{1}\left(\lambda^{*}\left(\tau, t_{g}, \ell\right)\right)=\varphi_{2}\left(\lambda^{*}\left(\tau, t_{g}, \ell\right)\right)=$ 0 . Then, solving $\Phi\left(\tau, t_{g}, \ell\right)=0$ with respect to $\ell$ in the neighborhood of $\left(\tau_{0}, t_{g 0}, 0\right)$ gives the homogeneous solution of $(34)$. For the particular solution, let $\lambda^{*}\left(\tau, t_{g}, \ell\right)=\left(\begin{array}{c}\eta^{*} \\ \bar{\alpha}\end{array}\right)$, where

$$
\begin{gathered}
\eta^{*}=\left(\tau_{0}^{-2}-m^{2} \tau^{-2}\right) \ddot{\bar{x}}-m^{2} \tau^{-2} \ddot{x}_{0}-x_{0}+\ell^{2}\left(-2 m^{-1} \tau V k-2 \bar{x}-2 x_{0}\right) \\
+C\left(\tau, t_{g}, \ell\right)-F\left(V+m \tau^{-1}\left(\dot{\bar{x}}+\dot{x}_{0}\right)\right)+F^{\prime}\left(V+\tau_{0}^{-1} \dot{x}_{0}\right) \cdot \tau_{0}^{-1} \overline{\bar{x}}, \\
\bar{\alpha}=\left(\begin{array}{c}
\alpha_{1} \\
\alpha_{2} \\
\alpha_{3} \\
\alpha_{4}
\end{array}\right)=\left(\begin{array}{c}
\ell^{2} \tau V T_{0} \\
V\left(\tau_{0}-m^{-1} \tau\right) \\
V\left(\tau t_{g}-\tau_{0} t_{g 0}\right)+\ell^{2} \tau V T_{0} \\
V\left(\tau_{0}-m^{-1} \tau\right)
\end{array}\right),
\end{gathered}
$$

It follows from the computation of $D_{\left(\tau, t_{g}\right)} \Phi\left(\tau_{0}, t_{g 0}, 0\right)$ that

$$
\varphi_{1}\left(\lambda^{*}\right)=V x_{02}\left(T_{0}\right)\left(\tau_{0}-m^{-1} \tau\right)+V\left(\tau_{0} t_{g 0}-\tau t_{g}\right)+x_{p, \eta^{*}}\left(T_{0}\right),
$$




$$
\begin{aligned}
\varphi_{2}\left(\lambda^{*}\right)=V \dot{x}_{02}\left(T_{0}\right)\left(\tau_{0}-m^{-1} \tau\right)+V\left(m^{-1} \tau-\tau_{0}\right)+ & \dot{x}_{p, \eta^{*}}\left(T_{0}\right) \\
& +\ell^{2} \tau V T_{0} \dot{x}_{01}\left(T_{0}\right)
\end{aligned}
$$

Since $m\left(t_{g}\right)=\frac{t_{g 0}-T_{0}}{t_{g}-T_{0}} \Rightarrow m^{\prime}\left(t_{g}\right)=-\left(t_{g 0}-T\right)^{-1}$ and for $\ell=0$, we have $C\left(\tau, t_{g}, \ell\right)=0$

$$
\begin{gathered}
\Rightarrow \partial_{\tau} \eta^{*}\left(\tau_{0}, t_{g}, 0\right)=F^{\prime}\left(V+\tau_{0}^{-1} \dot{x}_{0}\right)\left(-\tau_{0}^{-2}\left(\dot{\bar{x}}\left(\tau_{0}, t_{g 0}, 0\right)+\dot{x}_{0}\right)+\tau_{0}^{-1} \partial_{\tau} \dot{\bar{x}}\left(\tau_{0}, t_{g 0}, 0\right)\right) \\
-2 \tau_{0}^{-3}\left(\ddot{\bar{x}}\left(\tau_{0}, t_{g 0}, 0\right)+\ddot{x}_{0}\right)+F^{\prime}\left(V+\tau_{0}^{-1} \dot{x}_{0}\right) \cdot \tau_{0}^{-1} \partial_{\tau} \dot{\bar{x}}\left(\tau_{0}, t_{g 0}, 0\right) .
\end{gathered}
$$

Furthermore, since $\bar{x}\left(\tau_{0}, t_{g 0}, 0\right)=\dot{\bar{x}}\left(\tau_{0}, t_{g 0}, 0\right)=\ddot{\bar{x}}\left(\tau_{0}, t_{g 0}, 0\right)=0$, it follows that

$$
\partial_{\tau} \eta^{*}\left(\tau_{0}, t_{g}, 0\right)=-2 \tau_{0}^{-3} \ddot{x}_{0}+\tau_{0}^{-2} F^{\prime}\left(V+\tau_{0}^{-1} \dot{x}_{0}\right) \dot{x}_{0}
$$

Similarly,

$$
\begin{aligned}
& \partial_{t_{g}} \eta^{*}\left(\tau_{0}, t_{g 0}, 0\right) \\
& =F^{\prime}\left(V+\tau_{0}^{-1}\left(\dot{\bar{x}}\left(\tau_{0}, t_{g 0}, 0\right)+\dot{x}_{0}\right)\right)\left(-\tau_{0}^{-1}\left(t_{g 0}-T_{0}\right)^{-1}\left(\dot{\bar{x}}\left(\tau_{0}, t_{g 0}, 0\right)+\dot{x}_{0}\right)\right. \\
& \left.-\tau_{0}^{-1} \partial_{t_{g}} \dot{\bar{x}}\left(\tau_{0}, t_{g 0}, 0\right)\right)+F^{\prime}\left(V+\tau_{0}^{-1} \dot{x}_{0}\right) \cdot \tau_{0}^{-1} \partial_{t_{g}} \dot{\bar{x}}\left(\tau_{0}, t_{g 0}, 0\right) \\
& \quad+2 \tau_{0}^{-2}\left(t_{g 0}-T_{0}\right)^{-1}\left(\ddot{\bar{x}}\left(\tau_{0}, t_{g 0}, 0\right)+\ddot{x}_{0}\right) \\
& =2 \tau_{0}^{-2}\left(t_{g 0}-T_{0}\right)^{-1} \ddot{x}_{0}+F^{\prime}\left(V+\tau_{0}^{-1} \dot{x}_{0}\right) \tau_{0}^{-1}\left(t_{g 0}-T_{0}\right)^{-1} \dot{x}_{0} .
\end{aligned}
$$

Assuming the wronskian of the form $\omega=x_{01} \dot{x}_{02}-\dot{x}_{01} x_{02}$ and the particular solution

$$
x_{p, \eta}\left(T_{0}\right)=-\tau_{0}^{2} x_{01}\left(T_{0}\right) \int_{t_{g 0}}^{T_{0}} \frac{x_{02} \eta}{\omega} d k+\tau_{0}^{2} x_{02}\left(T_{0}\right) \int_{t_{g 0}}^{T_{0}} \frac{x_{01} \eta}{\omega} d k
$$

we have

$$
\begin{aligned}
\partial_{\tau} x_{p, \eta^{*}}\left(T_{0}\right)\left(\tau_{0}, t_{g 0}, 0\right) \\
=-\tau_{0}^{2} x_{01}\left(T_{0}\right) \int_{t_{g 0}}^{T_{0}} \frac{x_{02}}{\omega} \partial_{\tau} \eta^{*} d k+\tau_{0}^{2} x_{02}\left(T_{0}\right) \int_{t_{g 0}}^{T_{0}} \frac{x_{01}}{\omega} \partial_{\tau} \eta^{*} d k \\
=-\tau_{0}^{2} x_{01}\left(T_{0}\right) \int_{t_{g 0}}^{T_{0}} \frac{x_{02}}{\omega}\left[-2 \tau_{0}^{-3} \ddot{x}_{0}-\tau_{0}^{-2} F^{\prime}\left(V+\tau_{0}^{-1} \dot{x}_{0}\right) \dot{x}_{0}\right] d k \\
+\tau_{0}^{2} x_{02}\left(T_{0}\right) \int_{t_{g 0}}^{T_{0}} \frac{x_{01}}{\omega}\left[-2 \tau_{0}^{-3} \ddot{x}_{0}-\tau_{0}^{-2} F^{\prime}\left(V+\tau_{0}^{-1} \dot{x}_{0}\right) \dot{x}_{0}\right] d k \\
=\tau_{0}^{-1} x_{01}\left(T_{0}\right) \int_{t_{g 0}}^{T_{0}} \frac{x_{02}}{\omega}\left[-2 \ddot{x}_{0}-\tau_{0} F^{\prime}\left(V+\tau_{0}^{-1} \dot{x}_{0}\right) \dot{x}_{0}\right] d k
\end{aligned}
$$




$$
\begin{gathered}
-\tau_{0}^{-1} x_{02}\left(T_{0}\right) \int_{t_{g 0}}^{T_{0}} \frac{x_{01}}{\omega}\left[2 \ddot{x}_{0}+\tau_{0} F^{\prime}\left(V+\tau_{0}^{-1} \dot{x}_{0}\right) \dot{x}_{0}\right] d k, \\
\Rightarrow \partial_{\tau} x_{p, \eta^{*}}\left(T_{0}\right)\left(\tau_{0}, t_{g 0}, 0\right)=\tau_{0}^{-1} x_{01}\left(T_{0}\right) I_{1}-\tau_{0}^{-1} x_{02}\left(T_{0}\right) I_{2},
\end{gathered}
$$

where

$$
\begin{aligned}
& I_{1}=\int_{t_{g 0}}^{T_{0}} \frac{x_{01}}{\omega}\left[2 \ddot{x}_{0}+\tau_{0} F^{\prime}\left(V+\tau_{0}^{-1} \dot{x}_{0}\right) \dot{x}_{0}\right] d k, \\
& I_{2}=\int_{t_{g 0}}^{T_{0}} \frac{x_{02}}{\omega}\left[-2 \ddot{x}_{0}-\tau_{0} F^{\prime}\left(V+\tau_{0}^{-1} \dot{x}_{0}\right) \dot{x}_{0}\right] d k .
\end{aligned}
$$

Following the same procedure,

$$
\begin{aligned}
\partial_{t_{g}} x_{p, \eta^{*}}\left(T_{0}\right)\left(\tau_{0}, t_{g 0}, 0\right) & =-\tau_{0}^{2} x_{01}\left(T_{0}\right) \int_{t_{g 0}}^{T_{0}} \frac{x_{02}}{\omega}\left[\frac{2 \ddot{x}_{0}}{\tau_{0}^{2}\left(t_{g 0}-T_{0}\right)}\right. \\
& \left.+F^{\prime}\left(V+\frac{\dot{x}_{0}}{\tau_{0}}\right) \frac{\dot{x}_{0}}{\tau_{0}\left(t_{g 0}-T_{0}\right)}\right] d k \\
& +\tau_{0}^{2} x_{02}\left(T_{0}\right) \int_{t_{g 0}}^{T_{0}} \frac{x_{01}}{\omega}\left[\frac{-2 \ddot{x}_{0}}{\tau_{0}^{3}}-\frac{1}{\tau_{0}^{2}} F^{\prime}\left(V+\frac{\dot{x}_{0}}{\tau_{0}}\right) \dot{x}_{0}\right] d k . \\
\Rightarrow \partial_{t_{g}} x_{p, \eta^{*}}\left(T_{0}\right)\left(\tau_{0}, t_{g 0}, 0\right) & =-\frac{x_{01}\left(T_{0}\right)}{\left(t_{g 0}-T_{0}\right)} I_{2}+\frac{x_{02}\left(T_{0}\right)}{\left(t_{g 0}-T_{0}\right)} I_{1},
\end{aligned}
$$

Therefore, the partial derivatives of (36) and (37), respectively, becomes

$$
\begin{aligned}
& \partial_{\tau} \varphi_{1}\left(\tau_{0}, t_{g 0}, 0\right)=x_{02}\left(T_{0}\right) V+V t_{g 0}+\partial_{\tau} x_{p, \eta^{*}}\left(T_{0}\right)\left(\tau_{0}, t_{g 0}, 0\right), \\
& =x_{02}\left(T_{0}\right) V+V t_{g 0}+\tau_{0}^{-1} x_{01}\left(T_{0}\right) I_{2}-\tau_{0}^{-1} x_{02}\left(T_{0}\right) I_{1}, \\
& \partial_{\tau} \varphi_{2}\left(\tau_{0}, t_{g 0}, 0\right)=\dot{x}_{02}\left(T_{0}\right) V+V+\partial_{\tau} \dot{x}_{p, \eta^{*}}\left(T_{0}\right)\left(\tau_{0}, t_{g 0}, 0\right), \\
& =\dot{x}_{02}\left(T_{0}\right) V-V+\tau_{0}^{-1} x_{01}\left(T_{0}\right) I_{2}-\tau_{0}^{-1} x_{02}\left(T_{0}\right) I_{1}, \\
& \partial_{t_{g}} \varphi_{1}\left(\tau_{0}, t_{g 0}, 0\right)=-\frac{\tau_{0} V}{t_{g 0}-T_{0}} x_{02}\left(T_{0}\right)+V \tau_{0}+\partial_{t_{g}} x_{p, \eta^{*}}\left(T_{0}\right)\left(\tau_{0}, t_{g 0}, 0\right) \\
& =-\frac{\tau_{0} V}{t_{g 0}-T_{0}} x_{02}\left(T_{0}\right)-V \tau_{0}-\frac{x_{01}\left(T_{0}\right) I_{2}}{t_{g 0}-T_{0}}+\frac{x_{02}\left(T_{0}\right) I_{1}}{t_{g 0}-T_{0}}, \\
& \partial_{t_{g}} \varphi_{2}\left(\tau_{0}, t_{g 0}, 0\right)=-\frac{\tau_{0} V}{t_{g 0}-T_{0}} \dot{x}_{02}\left(T_{0}\right)+\frac{\tau_{0} V}{t_{g 0}-T_{0}}+\partial_{t_{g}} \dot{x}_{p, \eta^{*}}\left(T_{0}\right)\left(\tau_{0}, t_{g 0}, 0\right),
\end{aligned}
$$




$$
=-\frac{\tau_{0} V}{t_{g 0}-T_{0}} \dot{x}_{02}\left(T_{0}\right)-\frac{\tau_{0} V}{t_{g 0}-T_{0}}-\frac{\dot{x}_{01}\left(T_{0}\right) I_{2}}{t_{g 0}-T_{0}}+\frac{\dot{x}_{02}\left(T_{0}\right) I_{1}}{t_{g 0}-T_{0}} .
$$

Thus, the determinant of $D_{\left(\tau, t_{g}\right)} \Phi\left(\tau_{0}, t_{g}, 0\right)$ becomes

$$
\begin{aligned}
& \operatorname{det} D_{\left(\tau, t_{g}\right)} \Phi\left(\tau_{0}, t_{g}, 0\right)=\left(\partial_{\tau} \varphi_{1} \cdot \partial_{t_{g}} \varphi_{2}-\partial_{\tau} \varphi_{2} \cdot \partial_{t_{g}} \varphi_{1}\right)\left(\tau_{0}, t_{g}, 0\right)= \\
& \left(x_{02}\left(T_{0}\right) V+V t_{g 0}+\tau_{0}^{-1} x_{01}\left(T_{0}\right) I_{2}-\tau_{0}^{-1} x_{02}\left(T_{0}\right) I_{1}\right)\left[\begin{array}{c}
-\frac{\tau_{0} V}{t_{g 0}-T_{0}} \dot{x}_{02}\left(T_{0}\right)+\frac{\tau_{0} V}{t_{g 0}-T_{0}} \\
-\frac{\dot{x}_{01}\left(T_{0}\right) I_{2}}{t_{g 0}-T_{0}}+\frac{\dot{x}_{02}\left(T_{0}\right) I_{1}}{t_{g 0}-T_{0}}
\end{array}\right] \\
& -\left(\dot{x}_{02}\left(T_{0}\right) V-V+\tau_{0}^{-1} x_{01}\left(T_{0}\right) I_{2}-\tau_{0}^{-1} x_{02}\left(T_{0}\right) I_{1}\right)\left[\begin{array}{c}
-\frac{\tau_{0} V}{t_{g 0}-T_{0}} x_{02}\left(T_{0}\right)-\tau_{0} V \\
-\frac{x_{01}\left(T_{0}\right) I_{2}}{t_{g 0}-T_{0}}+\frac{x_{02}\left(T_{0}\right) I_{1}}{t_{g 0}-T_{0}}
\end{array}\right] \\
& =-\frac{\tau_{0} V^{2} t_{g 0} \dot{x}_{02}\left(T_{0}\right)+\tau_{0} V^{2} \dot{x}_{02}\left(T_{0}\right)-\tau_{0} V^{2}+\frac{\tau_{0} V^{2} t_{g 0}}{t_{g 0}-T_{0}}-\frac{V t_{g 0}}{t_{g 0}-T_{0}} \dot{x}_{01}\left(T_{0}\right) I_{2}}{+V \dot{x}_{01}\left(T_{0}\right) I_{2}+\frac{V t_{g 0}}{t_{g 0}-T_{0}} \dot{x}_{02}\left(T_{0}\right) I_{1}-\dot{x}_{02}\left(T_{0}\right) V I_{1},} \\
& =-\frac{\tau_{0} V^{2} T_{0}}{t_{g 0}-T_{0}} \dot{x}_{02}\left(T_{0}\right)+\frac{\tau_{0} T_{0}}{t_{g 0}-T_{0}}-\frac{V T_{0}}{t_{g 0}-T_{0}} \dot{x}_{01}\left(T_{0}\right) I_{2}+\frac{V T_{0}}{t_{g 0}-T_{0}} \dot{x}_{02}\left(T_{0}\right) I_{1} .
\end{aligned}
$$

Thus, det $D_{\left(\tau, t_{g}\right)} \Phi\left(\tau_{0}, t_{g 0}, 0\right) \neq 0$

$$
\Leftrightarrow-\tau_{0} V^{2} \dot{x}_{02}\left(T_{0}\right)+\tau_{0} V+\dot{x}_{02}\left(T_{0}\right) I_{1}-\dot{x}_{01}\left(T_{0}\right) I_{2} \neq 0
$$

Therefore, if (39) is satisfied, equation (33) can be solved using Implicit Function Theorem.

That means we can obtain $\left(\tau, t_{g}\right)$ in the neighborhood of

$$
\left(\tau, t_{g}, \ell\right)=\left(\tau_{0}, t_{g 0}, 0\right) \in \mathbb{R}^{3} .
$$

Hence, $x(t, \ell)=x_{0}(t)+\bar{x}\left(\tau(\ell), t_{g}(\ell), \ell\right)$ is the solution of $(1)$ in the neighborhood of $\left(x_{0}, \tau_{0}, 0\right)$. This concludes the proof of Theorem 3.7 and hence the existence of the solution for (1).

\section{Conclusion}

The global existence and uniqueness of wave solutions under spring-block model with non-smooth slip-dependent friction were proved using Poincare-Bendixson theroem and Lyapounov-Schmidt reduction technique. The non-smooth uncoupled version of the model was inclusion and the existence of its periodic solution 
was proved using Poincare-Bendixson theroem. However, the non-smooth coupled model was not an inclusion but a nonlinear differential equation which we used Lyapounov-Schmidt reduction technique to solve for both the periodic solution and the small perturbation solution that depended on parameter $\ell$. To derive the perturbation solution and hence use the Lyapounov-Schmidt reduction, the compatibility condition (39) needed to be satisfied. Thus, analytical solutions for non-smooth slide-dependent friction laws provided more insight to periodic waves and may help provide more understanding of earthquakes and other dissipative driven systems.

\section{References}

[1] J. M. Carlson, J. S. Langer, and BE. Shaw, 'Dynamics of earthquake faults', Reviews of Modern Physics, 66, 657, 1994.

[2] C. H. Scholz, The mechanics of earthquakes and faulting, Cambridge University Press, Cambridge, 1990.

[3] B. F. Feeny, A. Guran, N. Hinrichs, and K. Popp, A historical review of dry friction and stick-slip phenomena, Applied Mechanics Reviews 51 (5) 321-341(1998).

[4] C. H. Scholz, Earthquakes and friction laws, Nature, 391, 37-42 (1998).

[5] A. Ruina, Slip instability and state variable friction laws. J. Geophys. (1983) Res. 88, 10359-10370

[6] P. Okubo, Dynamic rupture modeling with laboratory-derived constitutive relations. J. Geophys. (1989) Res. 94, 12321-12335

[7] J. Dieterich, and B. Kilgore, Implications of fault constitutive properties for earthquake prediction. Proc. Natl. Acad. Sci. USA. 93, 3787-3794

[8] B. Kostrov, and S. Das, Principles of earthquake source mechanics, Cambridge University Press

[9] U. Galvanetto, Sliding bifurcations in the dynamics of mechanical systems with dry friction - remarks for engineers and applied scientists, J. Sound Vibr., 276, 121-139 (2004).

[10] J. H. Dieterich, Time-dependent Friction and the Mechanism of Stick-slip, Pure Appl. Geophys.116, 790-806(1978); Rice, J. R., and Ruina, A. L. Stability of Steady Frictional Slipping, J. Appl. Mech.50, 343-349(1983). 
[11] R. Burridge and L. Knopoff, Model and theoretical seismicity, Bull. Seism. Soc.Am. 57, 341-371 (1967).

[12] J. M. Carlson and J. S. Langer, Mechanical model of an earthquake fault, Phys. Rev. A 40, 6470-6484 (1989).

[13] B. Gutenberg and C.F. Richter, Seismicity of the Earth and Associated Phenomena, 2nd ed. (Princeton, N.J.: Princeton University Press, 1954), pages 17-19 ("Frequency and energy of earthquakes")

[14] K. Annan, Propagation of local and global smoothed periodic waves in a spring-block model. IJPAM (2012) Vol. 81, No. 3, 439-461

[15] V.B.Ryabov, and H.M.Ito, Multistability and chaos in a spring-block model, Phys. Rev. E, 52, No.6, P.6101- 6112(1995). 
